\title{
T-cell exhaustion limits immune reactivity and is associated with good prognosis in autoimmune disease
}

A transcriptional signature of CD8 T-cell exhaustion is associated with good prognosis in multiple autoimmune diseases-including anti-neutrophil cytoplasmic antibodyassociated vasculitis (AAV) and systemic lupus erythematosus (SLE) - but with poor outcomes in chronic infections, say researchers Eoin McKinney and colleagues. They suggest that measuring this signature could enable identification of patients at high risk of disease relapse and facilitate personalized therapy.

CD8 T-cell exhaustion was initially identified during chronic viral infection and occurs in response to persistence of antigen and a lack of co-stimulatory signals from CD4 T cells. Exhausted CD8 T cells are characterized by loss of effector function, persistent expression of inhibitory receptors and changes in gene expression that result in a transcriptional signature distinct from that of effector, memory or anergic T cells.

"The process of exhaustion limits the effectiveness of the $\mathrm{T}$-cell response to persistent infections and consequently results in poor outcomes as the virus persists in the host," explains McKinney. "We hypothesized that autoimmune disease represents an analogous state of immunity toward a persistent self-antigen, and reasoned that a similar process of T-cell exhaustion might limit immune reactivity in this context."

To investigate this hypothesis, the researchers analysed the transcriptomes of purified CD4 and CD8 T cells obtained from prospective cohorts of patients with active, untreated AAV or SLE. They then identified correlations between patterns of transcription and clinical outcomes, including time to relapse and relapse frequency, during a median follow-up of 5 years. "The prospective collection of detailed clinical information combined with sophisticated genome-wide transcriptomics on specific immune cell subsets was a key requisite of our approach. We chose to focus on the heterogeneity of the course of autoinflammatory disease, with the aim of better understanding the differences between patients with differing clinical outcomes, rather than compare patients with healthy controls," says McKinney.

The researchers found that a gene expression signature that reflects CD8 T-cell exhaustion, similar to that identified in chronic viral infection, was associated with low rates of AAV or SLE disease relapse during follow-up, but not with differences in disease activity at the time of sampling. By contrast, a type I interferon response signature was associated with disease activity, but not with long-term clinical outcomes. "These observations, supported by other work from our laboratory, suggest that disease activity and relapse are controlled by distinct processes and might ultimately require distinct treatment approaches," explains McKinney.

Further analyses of other patient cohorts using surrogate markers of transcriptional signatures of exhaustion and co-stimulation, such as expression of the transcriptional co-activator $K A T 2 B$, confirmed that T-cell exhaustion is associated with good prognosis in various autoimmune and inflammatory diseases (AAV, SLE, type 1 diabetes mellitus, idiopathic pulmonary fibrosis and dengue haemorrhagic fever), but with poor responses to therapy in patients with hepatitis $C$ virus infection and to vaccines against malaria, yellow fever and influenza.

Based on these findings, the researchers suggest that induction of T-cell exhaustion could be a potential therapeutic strategy for patients with autoimmune disease. A previous study showed that the process of T-cell exhaustion can be reversed by blocking inhibitory signals through the exhaustion-associated receptor PD-1. Conversely, McKinney and colleagues report that enhancing these signals using PD-1 ligand can induce exhaustion in vitro. "In principle,

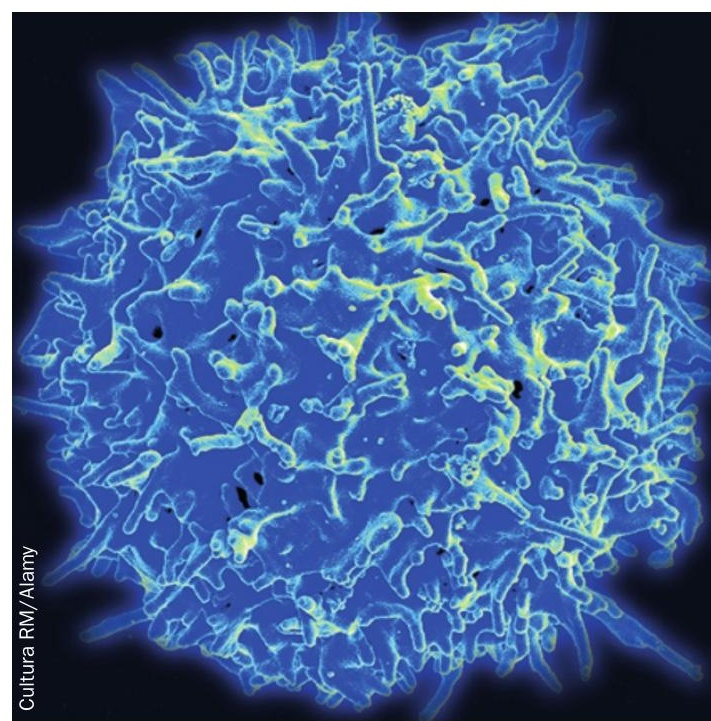

a transcriptional pattern associated with relapsing disease might be converted to one that is characteristic of good outcomes," concludes McKinney. "This approach might ultimately lead to novel targeted therapies for autoimmune and inflammatory diseases."

The researchers have developed a test based on quantitative PCR that can be used to identify the pattern of T-cell exhaustion in patient blood samples. They hope to make this test available to clinicians to enable them to predict the course of autoimmune disease in individual patients. In addition, they have begun a clinical trial to determine whether treatment choices informed by this test might improve clinical outcomes in patients with inflammatory bowel disease.

\section{Ellen F. Carney}

This article has also been published in Nat. Rev. Rheumatol. (doi:10.1038/nrrheum.2015.101).

\footnotetext{
Original article McKinney, E. F. et al.T-cell exhaustion, co-stimulation and clinical outcome in autoimmunity and infection. Nature doi:10.1038/nature14468

Further reading Barber, D. L. et al. Restoring function in exhausted CD8 T cells during chronic viral infection. Nature 439, 682-687 (2006)
} 